and make them purer and whiter, by preventing the smoke which at present mixes with our town fogs and aggravates their character, and prevents them dissolving when they enter our rooms. Smoke descends during a fog, because the smoke particles are good radiators, and soon get cooled and form nuclei on which the water vapour condenses. The smoke thus becomes heavier and falls. This explains why falling smoke is often a sign of coming rain. It indicates a saturated condition of the atmosphere.

Sulphur when burned has been shown to be an intensely active fog-producer. Calculation shows that there are more than 200 tons of sulphur burned with the coal every winter day in London, a quantity so enormous as quite to account for the density of the London fogs. It is suggested that some restriction ought to be put on the amount of sulphur in the coal used in towns.

Before utterly condemning the smoke and the sulphur, it was pointed out that it would be necessary thoroughly to investigate and fully to consider the value of smoke as a deodoriser, and also the powerful antiseptic properties of the sulphurous acid formed by the burning sulphur. The air during fogs is still and stagnant. There is no current to clear away the foul smells and deadly germs that float in the air, which might be more deadly than they are, were it not for the suspended soot and burned sulphur. We must therefore be on our guard lest we substitute a great and hidden danger for an evident but less evil.

\section{ON THE SPECTRUM OF CARBON}

A LTHOUGH fifteen years have passed since the possibility of one substance possessing more than one spectrum was first suggested by Pliicker and Hittorf the question of the existence of double spectra cannot yet be considered as decided. One of the clements to which multiple spectra bave been attributed is carbon, which was at one time supposed to possess four different spectra: of these one has been shown to be due to oxide of manganese, a second to oxides of carbon, the origin of a third (obtained only from oxides of carbon) has hardly been discussed (though it may prove to be one of the true carbon spectra), and the other "carbon" spectrum-the best known of all-is.the one first attributed to carbon by Attfield, but ascribed to acetylene by Ångström.

In a paper read before the Royal Society, and of which an abstract is given in NATURE, vol. xxii. p. 620, Professors Liveing and Dewar describe experiments to prove that this spectrum is that of a hydrocarbon, and not of carbon itself; and also that certain blue bands, best seen in the flame-spectrum of cyanogen, are due to compounds of carbon and nitrogen, and not to carbon itself. They attribute to hydrocarbon (amongst others) the yellowishgreen group, which we will call $\gamma$, of wave-lengths from about 5635 to 5478 , and the emerald-green group, which we will call $\delta$, of wave-lengths from about 5165 to 5082 ; and they attribute to nitro-carbon the two blue groups of wavelengths 4600 to 4502 and 4220 to 4158 , which we will call $\theta$ and $\zeta$ respectively.

As these result are directly opposed to my own experience, I have thought it necessary to repeat two of the experiments described in my paper on the carbon spectra in the Philosophical Magazine for October, 1869, under such conditions as to exclude (as far as lay in my power) all trace of hydrogen in the one case, and of nitrogen in the other.

The difficulty of supposing carbon to be present in the state of vapour at any temperature which we can command seems to be the chief reason why so many investigators think it necessary to attribute the spectrum in question (with experimental evidence or without it) to compounds of carbon. I am not aware that Angström ever gave any experimental proof of his assertion that this spectrum was caused by acetylene.
On the other hand, the evidence that the spectrum is due to carbon is that first stated by Attfield, that if these lines "are absent in flames in which carbon is absent, and present in flames in which carbon is present," if they are "observable equally in the flame of the oxide, sulphide, and nitride as well as in the hydride of carbon," and if "present whether the incandescence be produced by the chemical force, as in burning jets of the gases in the open air or by the electric force, as when hermeticallysealed tubes of the gases are exposed to the discharge of a powerful induction-coil," then they "must be due to incandescent carbon vapour" ; and if this is borne out by experiment the conclusion that the lines are due to carbon (as gas, liquid or solid) cannot be resisted, whatever may be the apparent impossibility of volatilising or even liquifying carbon, even by the most powerful current of electricity directed through it.

We must bear in mind how very small a quantity of a gas is often sufficient to give us a spectrum, and when the carbon spectrum is obtained by the decomposition of olefiant gas or cyanogen by passing sparks through the gas, the carbon certainly exists as gas in the compound which is decomposed, and before the liberated atoms unite together to form the molecules of the solid, there is surely no impossibility in their existing for the moment as gas-as gaseous carbon.

On an examination of Professors Liveing and Dewar's paper to ascertain the experimental evidence upon which the bands $\gamma$ and $\delta$ are attributed to hydrocarbon and not to carbon itself, we find it stated that "the green and blue bands characteristic of the hydrocarbon flame seem to be always present in the arcs, whatever the atmosphere. This is what we should expect if they be due, as Angström and Thalèn suppose, to acetylene, for the carbon electrodes always contain, even when they have been long heated in chlorine, a notable quantity of hydrogen."

Since then it is impossible to completely expel hydrogen from the carbon-poles, we must reject all the experiments in which the electric arc was observed in atmospheres of different gases, although "the green and blue hydrocarbon bands were seen more or less in all of them."

Turning then to other methods of producing the spectrum, we find it stated that in the flame of carefullydried cyanogen "the hydrocarbon bands were almost entirely absent" (they should have been entirely absent); "only the brightest green band was seen, and that faintly." Hence we are to infer, I suppose, that the bands $\gamma$ and $\delta$, so brilliant in the flame of cyanogen in air or oxygen, are due to the accidental presence of hydrogen (see the extract from Morren's paper, NATURE, vol. xxii. p. 7. Dibbits also speaks of this spectrum as "by far the most magnificent" he has seen).

Next we have the experiment of burning hydrocyanic acid, in which, as we have hydrogen present, we expect to find the hydrocarbon bands brilliantly developed. But we find the result stated as "very much the same as that of cyanogen." The flames of hydrogen and sulphide of carbon, and of hydrogen and carbonic oxide, do not give the hydrocarbon bands (their spectra being continuous); a mixture of hydrogen and carbon tetrachloride gives them faintly, and a mixture of hydrogen and chloroform gives them strongly.

In all this we have no proof of the point in question, nor even any special probability that the bands are due to hydrocarbon; and yet, in the face of experiments in which the spectrum is obtained from cyanogen, when care has been taken to exclude hydrogen, we are asked to attribute the bands to the hydrocarbon formed by combination with some trace of hydrogen (as water or otherwise), supposed to be present as impurity. In the same way the presence of the bands $\theta$ and $\zeta$ obtained under circumstances when nitrogen has been intentionally excluded, is to be explained by "the extreme difficulty of 
removing the last traces of air." So that in the case of cyanogen with a trace of hydrogen present, the spark persists in giving us the spectrum of hydrocarbon; and when we have naphthalin with a trace of nitrogen present, it gives us the spectrum of nitrocarbon !

Attfield states that the spectrum in question is obtained from pure dry cyanogen. "The ignition of the gases having been effected in air, it was conceivable that hydrogen, nitrogen, or oxygen had influenced the phenomena. To eliminate this possible source of error the experiments were repeated out of contact with air. A thin glass tube one inch in diameter and three inches long, with platinum wires fused into its sides and its ends prolonged by glass quills having a capillary bore, was filled with pure dry cyanogen, and the greater portion of this gas then removed by a good air-pump. Another tube was similarly prepared with olefiant gas. The platinum wires in these tubes were then so connected with each other that the electric discharge from a powerful induction-coil could pass through both at the same time. On now observing the spectra of these two lights $\mathrm{n}$ the simultaneous manner previously described, the

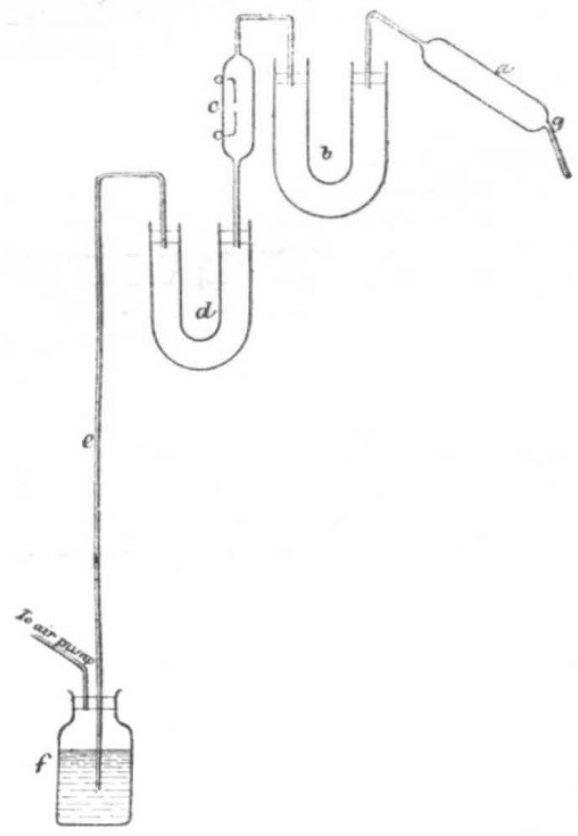

characteristic lines of the hydrocarbon spectrum were found to be rigidly continued in that of the nitrocarbon. Moreover, by the same method of simultaneous observation the spectrum of each of these electric flames, as they may be termed, was compared with the corresponding chemical flames, that is with the oxyhydrocarbon and oxynitrocarbon jets of gas burning in air. The characteristic lines were present in every case."

"The spectrum under investigation having then been obtained in one case when only carbon and hydrogen were present, and in another when all elements but carbon and nitrogen were absent, furnishes to my mind sufficient evidence that the spectrum is that of carbon."

Morren also adopted this method of producing the spectrum by taking the spark of an induction coil in a sufficiently rapid current of pure cyanogen at atmospheric pressure.

I have again repeated this experiment with cyanogen under conditions which would seem to ensure that the gas should be dry (see also Phil. Mag., 1875).

The cyanogen was prepared by heating pure cyanide of mercury, which was finely powdered and placed in a piece of combustion-tubing ( $a$ ) drawn out at both ends. In this it was repeatedly heated to the temperature of incipient decomposition whilst a current of dry air was drawn over it. One end of the tube was then closed by fusion at the point $g$, and the other bent round and fitted, as shown in the figure, to a U-tube $(b)$ containing phosphoric anhydride - the discharge-tube $c$ was interposed between this $U$-tube and a second U-tube $d$ also containing phosphoric anhydride, the other branch of which was connected to one end of a vertical tube $e$ of more than thirty inches in length, the lower end of which passed into mercury contained in the bottle $f$, the upper portion of which could be exhausted by means of the air-pump. The connections with the U-tube were made by means of perforated india-rubber stoppers, and the joints were surrounded during the experiment by melted paraffin.

The apparatus having been exhausted, the mercuric cyanide was heated till the apparatus was filled with cyanogen at atmospheric pressure; it was then again exhausted and again filled with cyanogen. After having been thus exhausted and re-filled five or six times, the spectrum of the spark between the wires at $c$ was examined at various pressures. The spectrum figured in my paper in the Philosophical Magazine for October, 1869, was obtained, the groups $\gamma$ and $\delta$, with which alone we are at present concerned, being the brightest in the whole spectrum. Next careful search was made for the red hydrogen line. The cross-wires of a one-prism spectroscope were accurately adjusted to the red line, as seen in a hydrogen vacuum tube, and the spectroscope was then directed upon the spark in the cyanogen. No trace of the line could be observed.

A second experiment was devoted to the examination of the spark in an atmosphere of naphthalin vapour, from which nitrogen had been excluded as far as possible, in order to ascertain whether the bands $\zeta$ and $\theta$, which Professors Liveing and Dewar attribute to cyanogen, would be produced. Professors Liveing and Dewar are somewhat in error in saying that I laid much stress on the occurrence of these bands in carbonic oxide. They were never obtained very brilliantiy from carbonic oxide (except under pressure), but they are obtained brilliantly from a naphthalin vacuum tube. I have obtained them also from a vacuum tube containing pure marsh-gas (my note-book remarks " $\theta$ very bright"), and as confirmation by an independent observer, I would remark that Pliicker maps them in the spectrum of a vacuum tube containing methyl.

The vacuum tube in this second experiment contained pure solid naphthalin fused on the sides of the tube; this was placed in position so that the upper end passed through one hole in an india-rubber stopper into a flask filled with carbon dioxide; a vertical tube of thirty inches length passed through the second hole in the stopper of the flask, and its lower end dipped below mercury. The whole of the vacuum tube except the lowest portion was surrounded by a wider tube containing melted paraffin.

When the apparatus had been arranged, the experiment was commenced by passing a rapid current of carbonic acid through the vacuum tube, so as to fill the flask and escape through the mercury. After passing the gas for a considerable time, the lower end of the tube was closed by fusion, the naphthalin all melted down into this end, where it was made to boil violently, while the paraffin was maintained at a temperature of about $220^{\circ} \mathrm{C}$. After the current of naphthalin vapour had lasted some time, the upper end of the tube was closed by fusion, the tube removed and cooled, and its spectrum examined. It gave a spectrum in which the groups $\zeta$ and $\theta$ were plainly seen.

It is to be hoped that some independent observer will repeat these experiments, so as finally to settle the question of the origin of these bands of what I must still call the "carbon" spectrum.
W. M. WATTS 